
\title{
Research Suare \\ Enhancement of the Photocatalytic Activity of ZnO Nanoparticles and the Relationship With Thermal Annealing Temperature
}

\section{F.H. Aragon ( $\sim$ fermin964@hotmail.com )}

Universidade de Brasilia https://orcid.org/0000-0001-5336-1131

\section{Research Article}

Keywords: ZnO nanoparticles, photocatalytic activity, ZnO exciton emission peak, thermal annealing in air atmosphere, photodegradation of methylene blue

Posted Date: September 29th, 2021

DOl: https://doi.org/10.21203/rs.3.rs-943311/v1

License: (9) This work is licensed under a Creative Commons Attribution 4.0 International License.

Read Full License 


\title{
Enhancement of the photocatalytic activity of $\mathrm{ZnO}$ nanoparticles and the
}

\section{relationship with thermal annealing temperature}

\author{
S. C. Andia-Huaracha ${ }^{1}$, L. M. Zapana-Cayo ${ }^{1}$, F. F. H. Aragón ${ }^{1 *}$, J. C. Romero Aquino ${ }^{1}$, J. A. H. Coaquira ${ }^{2}$, C. \\ D. Gonzales-Lorenzo ${ }^{1}$, J. S. Ayala-Arenas ${ }^{1}$, J. L. Solis ${ }^{3}$, P. C. Morais ${ }^{2,4}$ and D. G. Pacheco-Salazar ${ }^{1}$ \\ ${ }^{1}$ Universidad Nacional de San Agustin de Arequipa, Av. Independencia s/n, Arequipa, Peru \\ ${ }^{2}$ Universidade de Brasília, Instituto de Física, Núcleo de Física Aplicada, Brasília DF 70910-900, Brazil \\ ${ }^{3}$ Universidad Nacional de Ingeniería, Facultad de Ciencias, Lima, Peru \\ ${ }^{4}$ Universidade Católica de Brasília, Programa de Pós-Graduação em Ciências Genômicas e Biotecnologia, \\ Brasília DF 70790-160, Brazil
}

\begin{abstract}
The present study describes the synthesis, characterization, and photocatalytic activity (PCA) evaluation of $\mathrm{ZnO}$ nanoparticles (NPs) prepared via the polymeric precursor method in a first step, following annealing in the range of 500 to $900{ }^{\circ} \mathrm{C}$ in a second step. The formation of the wurtzite crystal structure is confirmed via $\mathrm{x}$-ray diffraction data analysis. Thermal annealing treatments in atmospheric air are carried out to control the crystallite mean size and physical properties. The UV-Vis spectroscopy measurements show a decreasing trend of the optical band gap of the as-fabricated $\mathrm{ZnO} \mathrm{NPs}$, from $\sim 3.4 \mathrm{eV}$ to $\sim 3.1 \mathrm{eV}$, as the annealing temperature increases. The PCA of the $\mathrm{ZnO}$ NPs against methylene blue dye revealed an increase in efficiency as the annealing temperature increases, reaching with a maximum performance for the sample annealed at $\sim 700{ }^{\circ} \mathrm{C}$. Our findings suggest that the PCA of the $\mathrm{ZnO}$ NPs does not show a straightforward dependence on the NPs' size, but it seems to be correlated with the density of defects, such as oxygen vacancies, which are modulated by the annealing temperature. In addition, thermoluminescence glow curves exhibit a well-defined peak located at $\sim 622 \mathrm{~K}$ for the as-prepared $\mathrm{ZnO}$ nanopowder. The peak intensity shows a decreasing trend with the annealing temperature up to $\sim 700{ }^{\circ} \mathrm{C}$. This result is correlated with the modulation of traps as the annealing temperature
\end{abstract}


increases, which seems to be directly related to the amount of trapped charges (electrons and holes), leading to a marked reduction of the PCA.

Keywords: $\mathrm{ZnO}$ nanoparticles, photocatalytic activity, $\mathrm{ZnO}$ exciton emission peak, thermal annealing in air atmosphere, photodegradation of methylene blue.

*Corresponding author ffharagon@gmail.com 


\section{Introduction}

Nowadays, a variety of colorants are introduced into water bodies such as rivers and oceans from industrial wastes, primarily as a result of the use of organic dyes in the textile industry that are disposed of in sanitary landfills without prior treatment [1]. Many industrial organic dyes are dangerous, carcinogenic, poisonous, and adversely affect human health, the environment, and aquatic ecosystems [2]. In this context, the scientific community is constantly working on developing methods that can improve water remediation [3]. Nanotechnology offers new approaches for handling wastewater due to the possibility of modifying the physical-chemical properties of the raw materials, thus improving the efficiency of water remediation as well as water purification. A very promising raw material is zinc oxide $(\mathrm{ZnO})$, which in their wurtzite phase is thermodynamically more stable at ambient conditions. This metal oxide is a transparent n-type semiconductor with direct and broad band gap energy (Eg) with values ranging from 3.1 to $3.3 \mathrm{eV}$ at room temperature [4]. Moreover, $\mathrm{ZnO}$ can be used for many applications due to its outstanding characteristics such as low cost, easy synthesis, non-toxicity, high structural and chemical stability and good optoelectronic properties. Within the extensive list of applications of $\mathrm{ZnO}$ one highlights its use as organic and hybrid solar cells [5], gas sensors [6], antibacterial surfaces [7], and photocatalysts [8]. Concerning their photocatalytic activity (PCA), several studies have focused on fine-tuning the PCA of the $\mathrm{ZnO}$ host matrix and, in this regard, we can highlight (i) the fact that the PCA of $\mathrm{ZnO}$ is altered by doping with metal/nonmetal atoms, such as in cobalt-doped $\mathrm{ZnO}$ nanostructures, which showed significantly higher PCA than pure $\mathrm{ZnO}$ [9]; (ii) variations in particle morphology and nanostructure, resulting in remarkable changes in the PCA [10] [11]. However, in both cases, the intrinsic defects such as oxygen vacancies $\left(\mathrm{V}_{\mathrm{O}}\right)$ and interstitial zinc $\left(\mathrm{Zn}_{\mathrm{i}}\right)$ are modified. These defects can capture charge carriers, thereby delaying the recombination process and participating in the absorption of $\mathrm{OH}^{-}$ ions and atmospheric $\mathrm{O}_{2}$, which are essential to improve the PCA, as they act as active centers at the interface between the catalyst surface and organic pollutants. In line with the observations mentioned above, Kayaci et al. studied experimentally the role of $\mathrm{V}_{\mathrm{O}}$ and $\mathrm{Zn}_{\mathrm{i}}$ on the PCA of $\mathrm{ZnO}$, showing a strong influence of such defects. Also, the authors suggested that $\mathrm{Zn}_{\mathrm{i}}$ is less efficient than $\mathrm{V}_{\mathrm{O}}$ in the PCA of the $\mathrm{ZnO}$. This is due to the 
accessibility of these defects via PCA [12]. Furthermore, a theoretical work showed that annealing temperatures can tune the intrinsic defects in the $\mathrm{ZnO}$ matrix [11]. One interesting result of the latter reports is that $\mathrm{Zn}_{\mathrm{i}}$ shows an annihilation temperature $(219 \mathrm{~K})$ very much lower than the $\mathrm{V}_{\mathrm{O}}(909 \mathrm{~K})$ [13]. This report suggests that predominantly $\mathrm{V}_{\mathrm{O}}$ is present in $\mathrm{ZnO}$-based materials subjected to annealing at temperatures above $219 \mathrm{~K}$. On the other hand, $\mathrm{ZnO}$ exhibits thermoluminescence (TL) response under a wide range of irradiation sources, making it a promising raw material for dosimetry applications [14]. Besides, the TL response can also be used as a complementary characterization tool technique to estimate activation energies of trap levels inside the band gap. It can be useful to understand the improvement of the PCA, as it will be discussed later on in this report.

In the present study, $\mathrm{ZnO}$ nanopowders are synthesized by the polymer precursor chemical method and thermally annealed (TA) at different temperatures in air condition in order to improve the samples' crystallinity and modulate the mound of intrinsic defects. The effects of the annealing temperature on the structural and optical properties will be comprehensively discussed. The PCA of the $\mathrm{ZnO}$ nanopowders are assessed in the TA samples and the results are discussed in light of the experimental findings.

\section{Experimental Details}

$\mathrm{ZnO}$ nanopowders were synthesized by the polymer precursor chemical method using zinc nitrate hexahydrate $\left(\mathrm{Zn}\left(\mathrm{NO}_{3}\right)_{2} \bullet 6 \mathrm{H}_{2} \mathrm{O}\right)$, ethylene glycol $\left(\mathrm{C}_{2} \mathrm{H}_{4}(\mathrm{OH})_{2}\right)$, and citric acid $\left(\mathrm{C}_{6} \mathrm{H}_{8} \mathrm{O}_{7}\right)$ as reactants. In the synthesis, citric acid (47.7 wt \%) and zinc nitrate hexahydrate (31.7 wt \%) were initially dissolved in ethylene glycol (20.6 wt \%) at $\sim 70{ }^{\circ} \mathrm{C}$, under magnetic stirring. After complete dissolution, the temperature was raised up to $120{ }^{\circ} \mathrm{C}$ to promote citric acid and ethylene glycol polymerization. Then, the resin yield (mass of $\mathrm{ZnO}$

produced/mass of gel) was determined by annealing a known mass of the resin at $400{ }^{\circ} \mathrm{C}$ for 5 h. The resulting dry and brittle powder was grinded and calcined at $400{ }^{\circ} \mathrm{C}$ for $12 \mathrm{~h}$ to enhance the elimination of organic compounds, resulting in $\mathrm{ZnO}$ nanoparticles (NPs). The synthesized samples were labeled $\mathrm{ZnO}-400^{\circ} \mathrm{C}$ (asprepared), $\mathrm{ZnO}-500^{\circ} \mathrm{C}, \mathrm{ZnO}-600^{\circ} \mathrm{C}, \mathrm{ZnO}-700^{\circ} \mathrm{C}, \mathrm{ZnO}-800^{\circ} \mathrm{C}$, and $\mathrm{ZnO}-900^{\circ} \mathrm{C}$ as a result of the annealing 
temperatures, which ranged from 400 to $900{ }^{\circ} \mathrm{C}$ in air atmosphere. X-ray diffraction (XRD) measurements were performed using a MiniFlex 600 X-ray Diffractometer from Rigaku with $\mathrm{Cu} \operatorname{K} \alpha$ radiation $(\lambda=1.5418 \AA)$. Experiments were carried out in the range of $20^{\circ} \leq 2 \theta \leq 80^{\circ}$, and the diffraction patterns were analyzed using the Rietveld refinement method implemented in the Generalized Structure Analysis System (GSAS) software [15]. UV-Vis spectra were collected using a Shimadzu UV-1800 UV/Visible Scanning Spectrophotometer. Fourier-transform infrared spectroscopy (FTIR) was performed using the KBr pellet method in the $450-4000$ $\mathrm{cm}^{-1}$ range employing a Thermo Scientific (Nicola i10) spectrometer. The thermal decomposition behavior of the resin was studied by thermogravimetric analysis (TGA) and differential scanning calorimeter (DSC) with a PerkinElmer STA8000®. The data were recorded in the range of $30{ }^{\circ} \mathrm{C}-900{ }^{\circ} \mathrm{C}$, in $\mathrm{N}_{2}$ flow, with a heating rate of $10^{\circ} \mathrm{C} / \mathrm{min}$. The irradiation for thermoluminescence (TL) measurements of the samples was carried out at the Professional School of Physics of UNSA (EPF-UNSA) using a ${ }^{60}$ Co source from Picker, model Gammatron with a dose rate of $97.3 \mathrm{mGy} / \mathrm{min}$ at $10 \mathrm{~cm}$ from the source. The $\gamma$-irradiation was performed at room temperature and under conditions of electronic equilibrium. After irradiation the samples were kept in the dark until TL measurements. To evaluate the PCA of the samples, a methylene blue (MB) aqueous solution (3 ppm) was utilized; the samples were placed in solution at a concentration of $1 \mathrm{mg} / \mathrm{mL}$, following ultrasonication to promote powder disaggregation while providing homogeneous dispersions. Finally, the solution with the suspended NPs was exposed to UV irradiation using a UV-C as a light source. $3 \mathrm{~mL}$ of each sample was taken every 60 min during photodegradation reaction, being immediately covered with aluminum foil for further analyzis using the UV-Vis spectrophotometer.

\section{Results and discussion}

\subsection{Thermogravimetric analysis}

Figure 1 depicts the weight-loss curve, acquired by thermogravimetric analysis (TGA), during the synthesis of the ZnO NPs. Three distinct weight-loss steps are observed: (i) the first one develops between 23 
and $\sim 160{ }^{\circ} \mathrm{C}$ ( $\sim 24 \%$ weight-loss), which is related to the elimination of water and ethylene glycol; (ii) the second weight-loss step is assigned to the combustion of citric acid and nitrate and develops between $\sim 160$ and $\sim 350{ }^{\circ} \mathrm{C}$ ( $\sim 62 \%$ weight-loss), and (iii) the third step, running from $\sim 350$ to $\sim 500{ }^{\circ} \mathrm{C}$, occurs due to the formation of stable $\mathrm{ZnO}$ NPs and decomposition and burning of residual carbon components. A significant improvement in crystallinity is observed above $\sim 500{ }^{\circ} \mathrm{C}$.

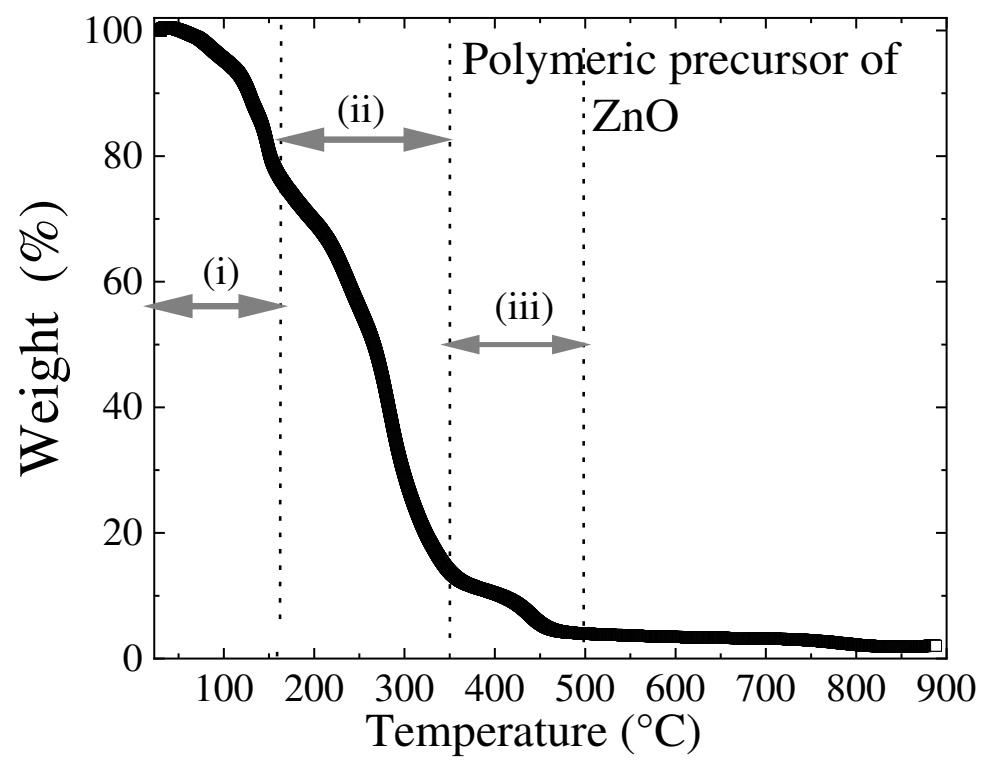

Figure 1 Thermogravimetric analysis curve obtained during the synthesis of the $\mathrm{ZnO}$ nanopowder.

\subsection{X-ray diffraction}

Figure 2 (a) shows the XRD patterns of the $\mathrm{ZnO}$ nanopowders subjected to the $\mathrm{TA}$ and after the methylene blue (MB) degradation protocol. As observed, all diffraction patterns show Bragg reflections consistent with the wurtzite structure (space group P63mc). The full width at half maximum (FWHM) of the Bragg reflections tend to decreases with the annealing temperature as shown in Fig 2 (a), implying an increase in the mean particle size and/or a reduction in the residual strain. All diffraction patterns (before and after photodegradation using MB dye) were refined using the Rietveld refinement method in order to determine the structural parameters (lattice constant, unit cell volume, and mean crystallite size). Figure 2 (b) shows a typical 
refinement carried out in the $\mathrm{ZnO}-500^{\circ} \mathrm{C}$ sample. Table 1 displays the lattice constants of the samples before and after the photodegradation protocol. The data listed in Table 1 show no drastic changes while comparing both sets of samples. Also, the evaluated lattice constants show larger values than that expected for bulk $\mathrm{ZnO}$ wurtzite structure [16], with no obvious tendency (see Table 1). This finding suggests a degree of structural disorder in all samples. However, the $c / a$ ratio displays a clear tendency to decrease as the annealing temperature is increased in both sets of samples (before and after the photodegradation protocol using MB) as collected in Fig. 2 (c). In this regard, our finding suggests that the unit cell is enlarged along the c-axis for the as-prepared samples, and it tends to the bulk wurtzite structure value $(c / a \sim 1.602)$ as the annealing temperature is increased. This result suggests the occurrence of likely strained states for the as-prepared samples, which become relaxed as the annealing temperature increases.

Table 1 Structural parameter of the ZnO NPs, which were annealed at different temperatures before and after the photodegradation using the MB dye. $S$ is the goodness of fit index ( $S=R w p / R e x p e c t e d)$.

\section{Before}

After

$\mathrm{TA}\left({ }^{\circ} \mathrm{C}\right)$

$a(\AA)$

$c(\AA)$

$V\left(\AA^{3}\right)$

S

$a(\AA)$

$c(\AA)$

$V\left(\AA^{3}\right)$

S

\section{As-}

prepared

500

600

700

800

900

3.2530

3.2532

3.2524

3.2533

3.2546

3.2533
5.2156

47.798

1.26

3.2532

5.2161

47.807

1.26

5.2128

47.777

1.3

3.2532

5.2129

47.779

1.31

5.2101

47.729

1.35

3.2537

5.2126

47.791

3.2534

5.2101

47.757

$5.2095 \quad 47.765$

5.2106

47.798

1.29

3.2538

47.728

1.36

3.2538

5.2083

47.755

1.32

$* a=b=3.250 \AA, c=5.207 \AA$, and $V=47.566 \AA^{3}$ for the bulk $\mathrm{ZnO}$ structure [16]. 

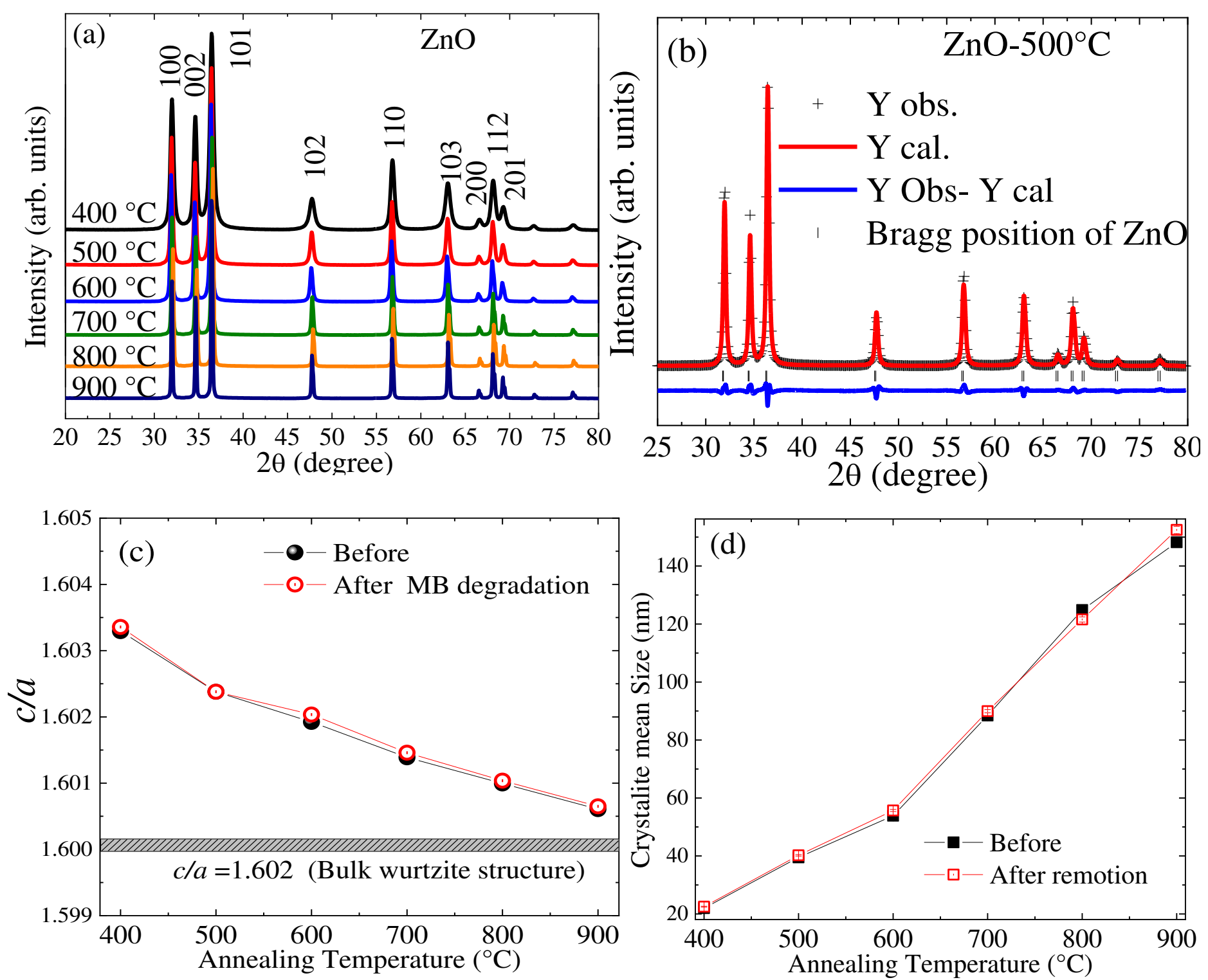

Figure 2 (a) X-ray diffraction patterns of the $\mathrm{ZnO}$ powders as-synthesized $\left(400{ }^{\circ} \mathrm{C}\right)$ and annealed between 500 and $900{ }^{\circ} \mathrm{C}$. (b) Rietveld refinement of the pattern corresponding to the sample annealed at $500{ }^{\circ} \mathrm{C}$. Symbols and solid lines indicate the experimental and calculated data, respectively. The blue solid line in the bottom represents the difference between experimental and calculated data. (c) c/a ratio as a function of the annealing temperature. (d) Mean crystallite size as a function of the annealing temperature.

The Scherrer relation: $\langle D\rangle=k \lambda / \beta \cdot \cos (\theta)$ is used to estimate the mean crystallite size $\langle D\rangle$, where $k$ is a constant ( $k=0.9$ for spherical particles), $\lambda$ is the wavelength of the $\mathrm{Cu}-\mathrm{K} \alpha$ radiation, $\beta$ is the full width at half maximum (FWHM) of the Bragg reflection, and $\theta$ is the corresponding diffraction angle. As observed in 
Fig. 2 (d), the mean particle size increases monotonically as the annealing temperature increases. Similar results were observed for the ZnO NPs after the photodegradation process. These findings are significant once they indicate that the $\mathrm{ZnO}$ NPs can be reused for photodegradation of MB dye several times without affecting their structural properties.

\subsection{UV-visible absorption spectroscopy}

In order to study the optical properties of the as-prepared and annealed samples, UV-Vis absorption spectra, in transmission mode, were recorded in the region from 250 to $850 \mathrm{~nm}$. As shown in Fig. 3 (a), an absorption band centered at $365 \mathrm{~nm}(3.39 \mathrm{eV})$ was observed. According to the literature, this peak is a characteristic feature of the wurtzite $\mathrm{ZnO}$ structure and it can be likely assigned to free excitons [11]. The observation of free excitons reflects the high crystallinity quality of the as-prepared $\mathrm{ZnO}$ NPs. The peak position allows one to estimate the band gap energy, $E_{g}=h c / \lambda=1240 / \lambda(\mathrm{nm})$, where $h$ is the Planck's constant, $c$ is the speed of light and $\lambda$ is the wavelength of maximum absorption [17]. As the annealing temperature increases, the position of the exciton peak shifted to higher wavelengths (lower energy), which implies a band gap closing as the annealing temperature is increased (see Fig. 3 (b)). The latter observation could be associated with the relaxation process, once the as-grown sample shows larger lattice constants and higher $c / a$ value (see Fig. 2 (c)). It is worth mentioning that the relaxation could be related to the decrease of oxygen vacancies in the samples upon annealing at higher temperatures, in air atmosphere, as reported in the literature [18]. This result is in agreement with a theoretical work, using density-functional theory to show that the band-gap of $\mathrm{ZnO}$ will decrease (increase) with increasing (decreasing) of the tensile (compressive) uniaxial strain [19]. Moreover, the peak observed in the absorption spectra of the as-prepared ZnO NPs is redshifted and becomes weaker as the annealing temperature increases. This finding seems to be related to a broad particle size distribution, as reported in the literature [20]. 

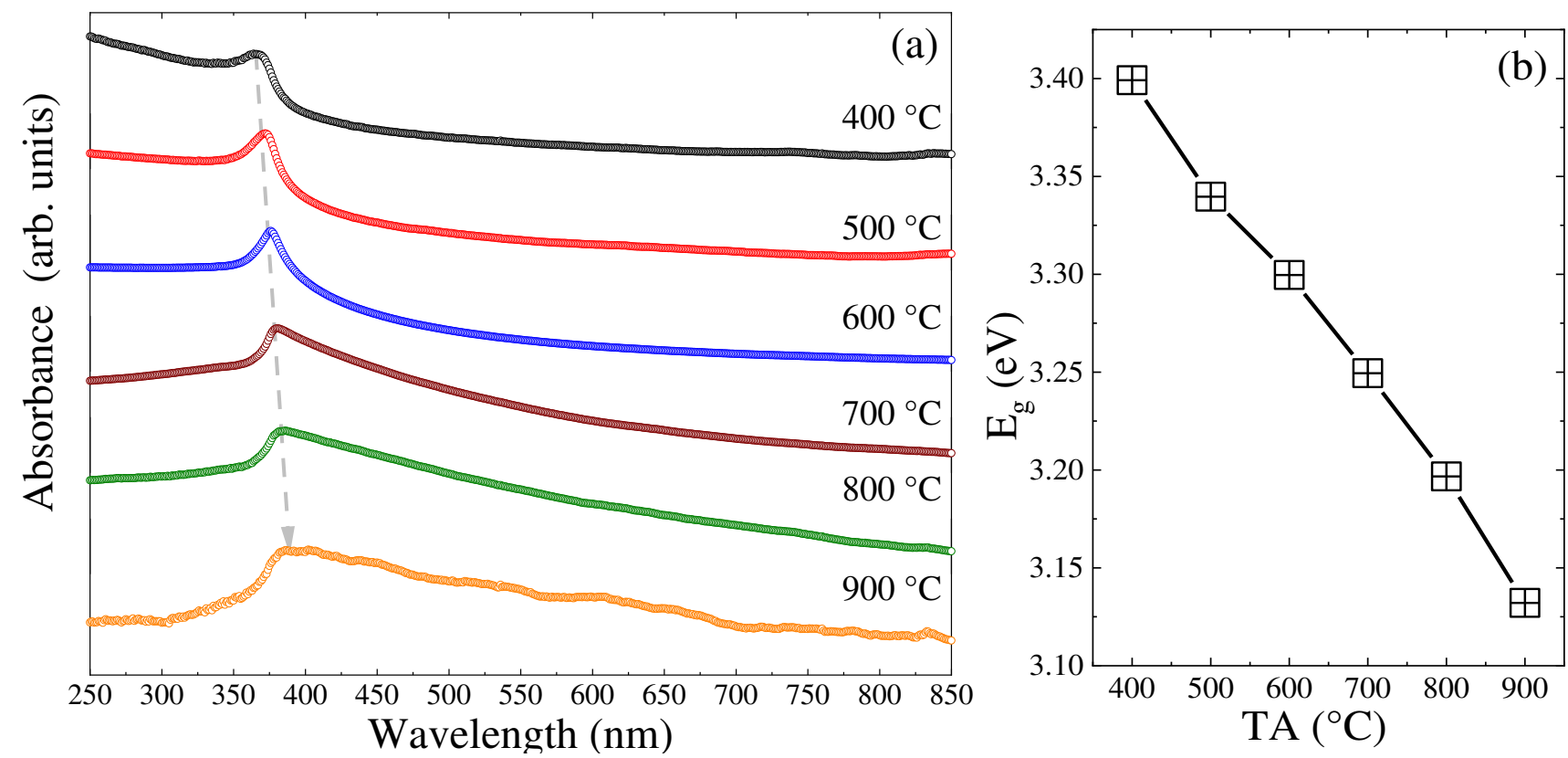

Figure 3 (a) UV-Vis spectroscopy (absorbance mode) spectra for the as-prepared $\left(400{ }^{\circ} \mathrm{C}\right.$ ) and the thermally annealed samples. The peak is identified as an excitonic peak. (b) Dependence of the band gap energy as a function of the annealing temperature.

\subsection{Fourier transform infrared spectroscopy}

The FTIR spectra of the as-prepared and annealed ZnO NPs are collected in Fig. 4. Here, the spectrum of the as-prepared sample shows a vibrational mode located at $3440 \mathrm{~cm}^{-1}$, which is assigned to $\mathrm{O}-\mathrm{H}$ stretching groups of water adsorbed onto ZnO NPs. This peak becomes weaken as the annealing temperature increases. It seems to be consistent with the total surface area reduction as the particle size becomes larger. Furthermore, the vibrational modes located at $\sim 2920 \mathrm{~cm}^{-1}$ and $\sim 2853 \mathrm{~cm}^{-1}$ are associated with the $\mathrm{C}-\mathrm{H}$ stretching vibrations. The band peaking at $1635 \mathrm{~cm}^{-1}$ corresponds to the $\mathrm{C}=\mathrm{O}$ symmetric stretching mode, meanwhile the band located at $1395 \mathrm{~cm}^{-1}$ corresponds to $\mathrm{C}=\mathrm{O}$ asymmetric stretching mode. The band observed at $875 \mathrm{~cm}^{-1}$ is assigned to the formation of tetrahedral coordination of $\mathrm{Zn}$, according to the literature [21]. The mode at 431 $\mathrm{cm}^{-1}$ is related to the $\mathrm{Zn}-\mathrm{O}$ stretching vibrations. It is worth noting that this is the more intense band and seems to be related to the excellent crystallinity of the samples, in good agreement with the results discussed above. To 
determine the effects of the MB photodegradation, mainly at the NPs' surface, FTIR measurements were carried out after the MB photodegradation process. Results are included in Fig. 4. From the spectra, no changes in the peak position of the $\mathrm{Zn}-\mathrm{O}$ stretching vibrations after the $\mathrm{MB}$ photodegradation is observed, suggesting that these materials can be reused without affecting the crystallinity of the $\mathrm{ZnO}$-based material. However, the intensity of the peak assigned to the $\mathrm{C}-\mathrm{H}$ and $\mathrm{C}=\mathrm{O}$ bonds shows a slight increase, which suggests the adsorption of MB molecules onto the NPs' surface.
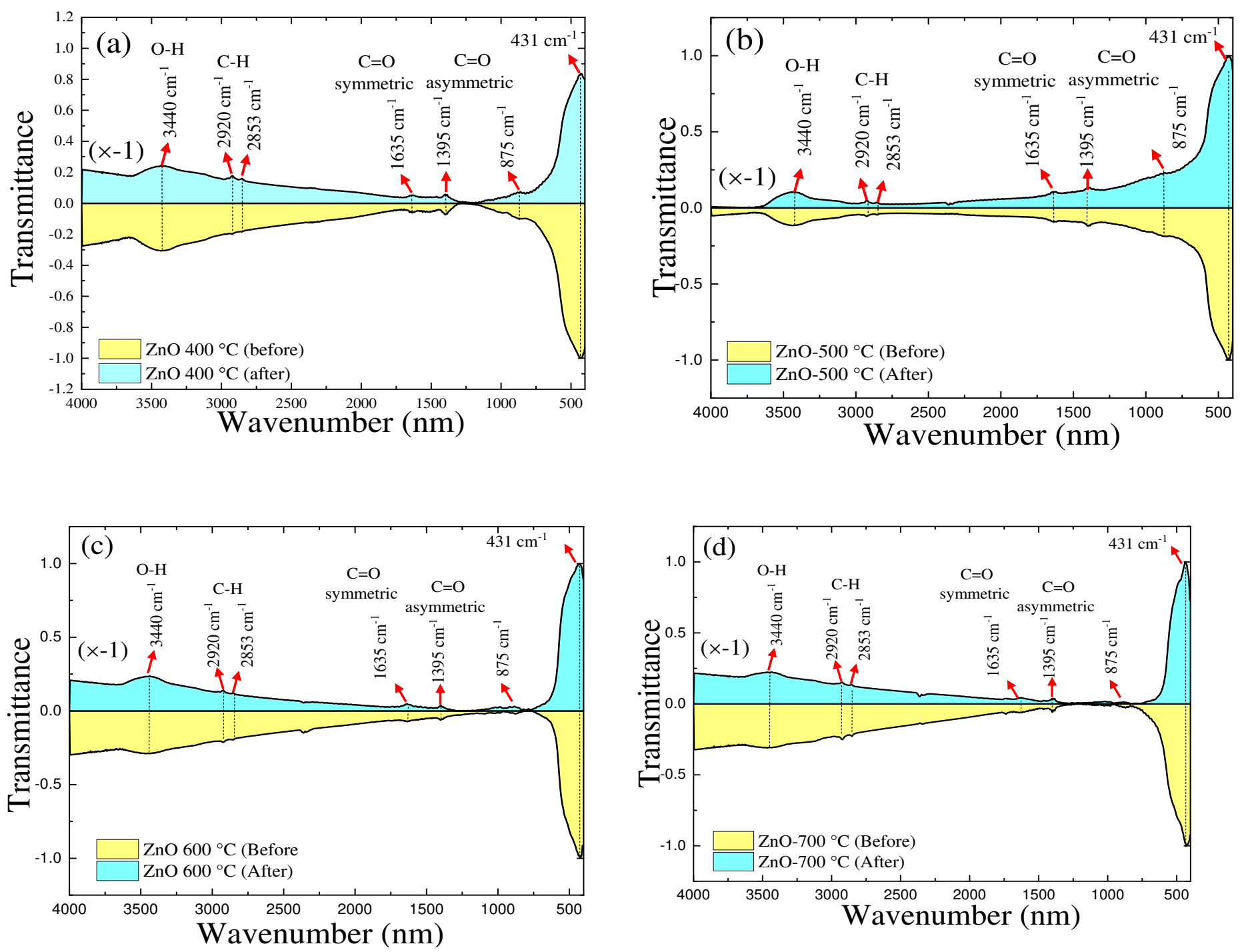

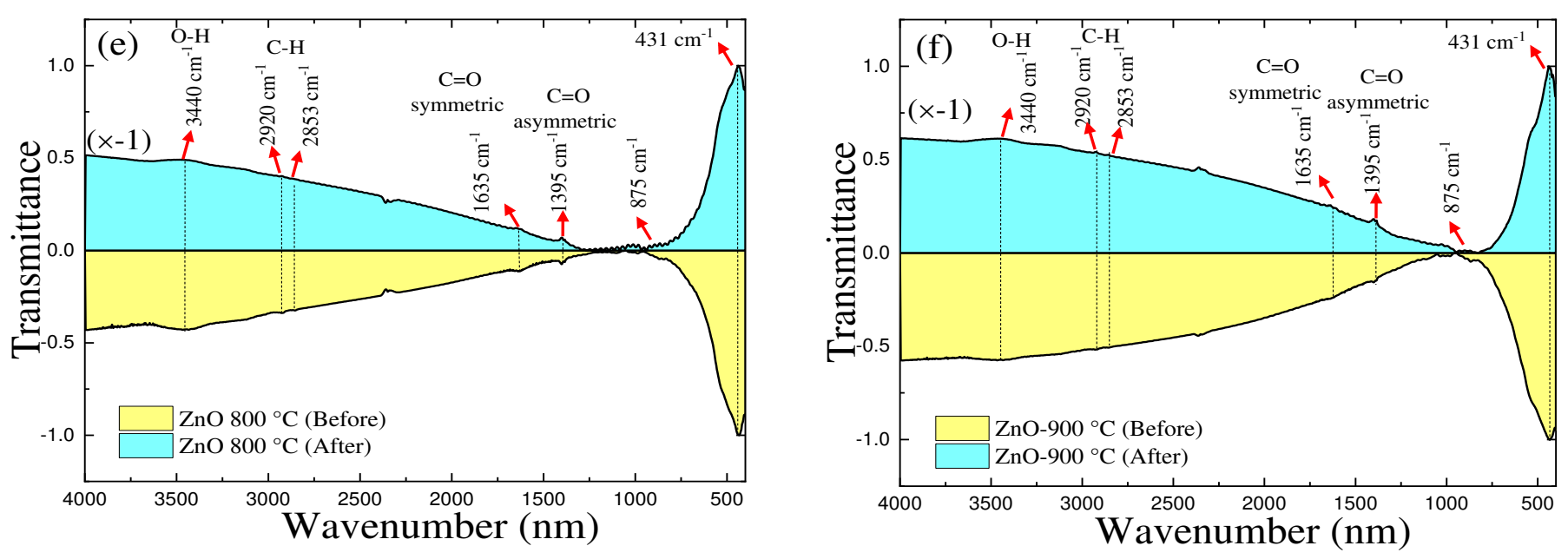

Figure 4. FTIR spectra of the as-prepared and annealed ZnO NPs were recorded before and after the photodegradation of the MB dye. (a) as-prepared, (b) $500{ }^{\circ} \mathrm{C}$, (c) $600{ }^{\circ} \mathrm{C}$, (d) $700{ }^{\circ} \mathrm{C}$, (e) $800{ }^{\circ} \mathrm{C}$, and (f) $900{ }^{\circ} \mathrm{C}$.

\subsection{Study of the photodegradation of methylene blue dye}

MB dissolved in distilled water was used to explore the PCA of the as-fabricated $\mathrm{ZnO}$ nanopowders. In order to determine the linear response region, UV-Vis absorption spectra of water solution of MB, varying the concentration from 0.5 to 5 ppm, were recorded and presented in Fig. 5 (a). As observed, the spectra exhibit two pronounced absorption bands at $292 \mathrm{~nm}$, which is related to the $\pi-\pi^{*}$ bond of the benzene ring and at $664 \mathrm{~nm}$, related to the $n-\pi^{*}$ bond (where $n$ is the free doublet of the nitrogen atom in the $\mathrm{C}=\mathrm{N}$ bond and free doublet of $\mathrm{S}$ atom in the $\mathrm{S}=\mathrm{C}$ bond). Near to the most intense peak (at $664 \mathrm{~nm}$ ) a shoulder peak at $615 \mathrm{~nm}$ is observed, which corresponds to the 0-1 vibronic transition [22]. Also, absorption bands located at 246 and $325 \mathrm{~nm}$ are determined near to the benzene ring band. The absorbance intensity of the $n-\pi^{*}$ band as a function of the MB concentration is shown in Fig. 5 (b). As observed, a linear behavior is determined in Fig. 5 (b), indicating that this band can be used to evaluate the photodegradation of the MB dye. We used the $3 \mathrm{ppm} \mathrm{MB}$ solution for the study of photodegradation. The concentration of the ZnO NPs was fixed to $1 \mathrm{mg} / \mathrm{mL}$. 

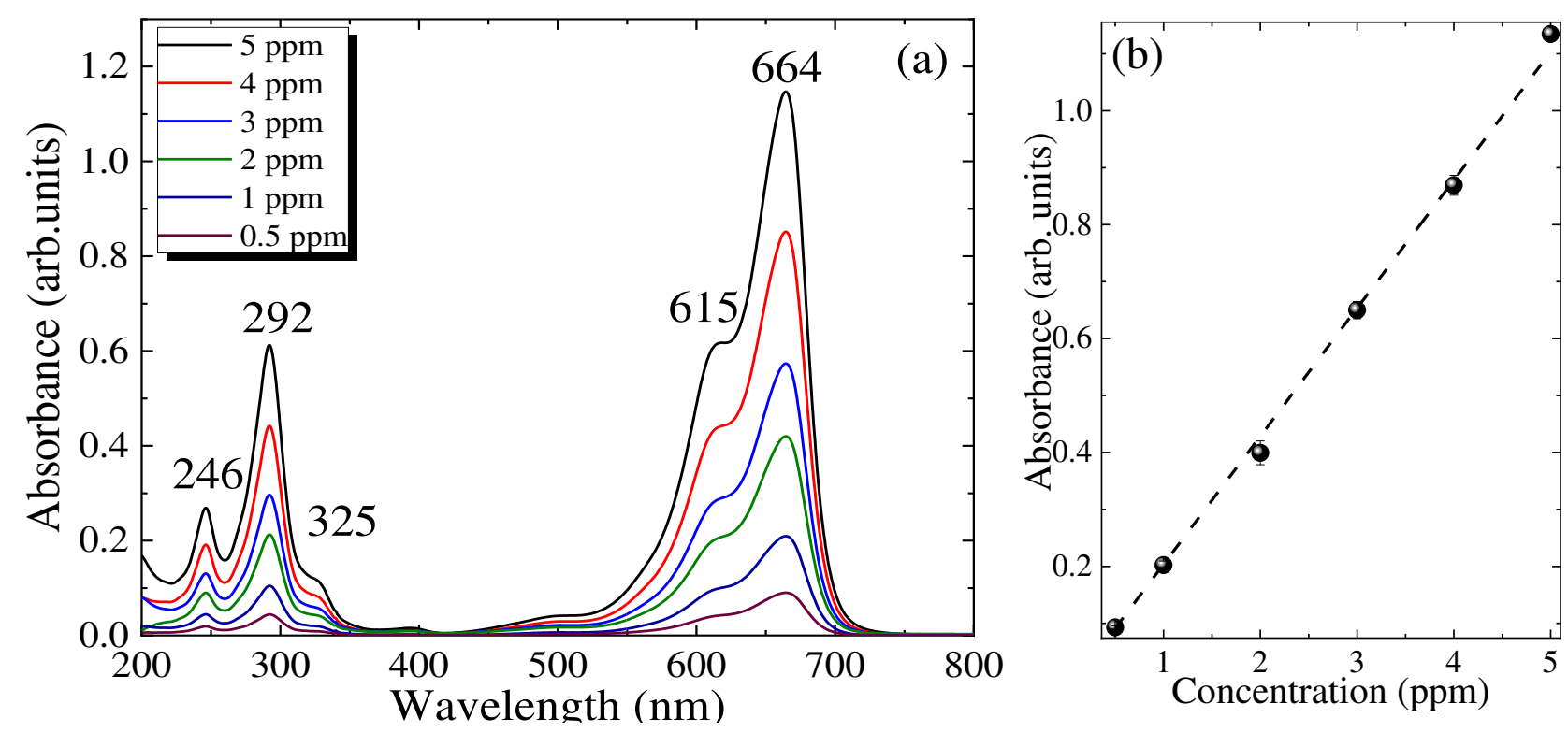

Figure 5 (a) UV-Vis absorption spectra of MB in water solution at different concentrations (from 0.5 to 5 ppm). (b) The absorbance intensity of the $n-\pi^{*}$ mode as a function of the MB concentration, showing a linear behavior.

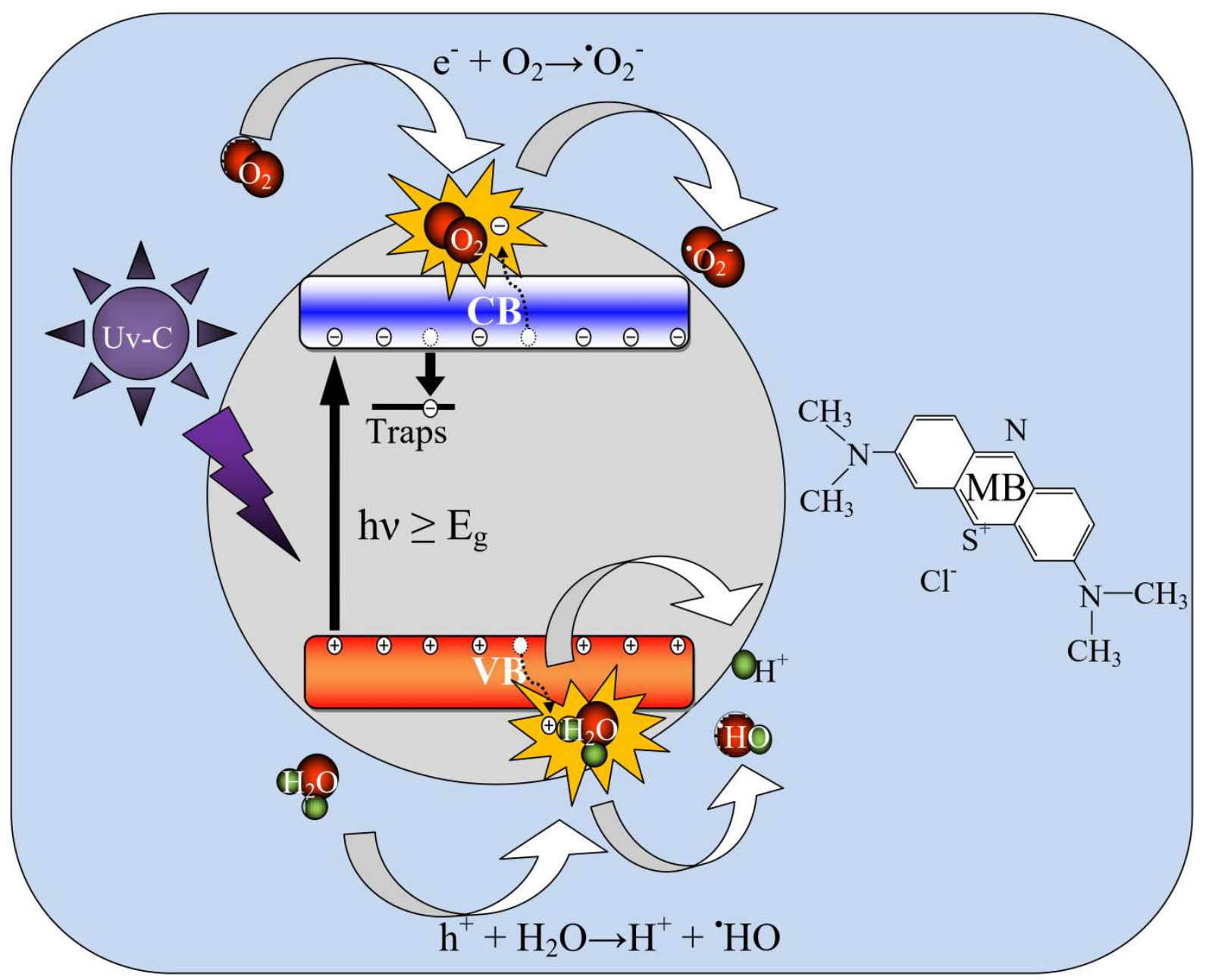


Figure 6. Schematic representation of the UV-C light interaction with ZnO NPs. The resultant electron-hole pairs produce reactive hydroxyl radicals $\left({ }^{\circ} \mathrm{OH}\right)$ and superoxide anion radicals $\left({ }^{\circ} \mathrm{O}_{2}^{-}\right)$in the valence band $(\mathrm{VB})$ and conduction band (CB), respectively, which degraded MB molecules. The amount of traps (evidenced through TL measurements), tuned by the thermal annealing, lead to a marked reduction of the PCA.

Figures 7 (a)-(c) show the UV-Vis absorbance of three MB dye solution containing zinc oxide NPs (annealed at 500, 700, and $900{ }^{\circ} \mathrm{C}$ ). The spectra were taken at different times of UV-C light exposure. As observed, the peak intensity of the adsorption band shows a decreasing trend as the exposure time increases, mainly during the first minutes. The intensity reduction can be explained as follow: the UV-C light leads to the generation of electron-hole $\left(\mathrm{e}^{-}-\mathrm{h}^{+}\right)$pairs in the conduction and valence bands of the $\mathrm{ZnO}$ NPs (which migrate to the nanoparticle surface), as it is schematically represented in Fig. 6. The holes interact with hydroxyl groups $\left(\mathrm{OH}^{-}\right)$(see Equation 1). Light irradiation also leads to the dissociation of water molecules in solution to produce highly reactive hydroxyl radicals $\left({ }^{\circ} \mathrm{OH}\right)$ (see Equation 2$)$. The electrons interact with oxygen molecules $\left(\mathrm{O}_{2}\right)$ to form the superoxide anion radicals $\left({ }^{\circ} \mathrm{O}_{2}^{-}\right)$(see Equation 3). In agreement with the following equations [9]:

$$
\begin{gathered}
\mathrm{h}^{+}+\mathrm{OH}^{-} \rightarrow{ }^{\circ} \mathrm{OH} \\
\mathrm{h}^{+}+\mathrm{H}_{2} \mathrm{O} \rightarrow \mathrm{H}^{+}+{ }^{\cdot} \mathrm{OH} \\
\mathrm{e}^{-}+\mathrm{O}_{2} \rightarrow{ }^{\circ} \mathrm{O}_{2}^{-}
\end{gathered}
$$

The reactive species ${ }^{\circ} \mathrm{OH}$ and ${ }^{\circ} \mathrm{O}_{2}^{-}$photocreated in the aqueous medium react with the dissolved MB molecules and break down them to produce $\mathrm{CO}_{2}$ and $\mathrm{H}_{2} \mathrm{O}$ molecules.

To determine the quantitative $\mathrm{MB}$ photodegradation at different times, we related the absorption intensity with the $\mathrm{MB}$ concentration $(C)$ in the evaluated suspension. Accordingly, the following equation $\left(C-C_{0}\right) / C_{0} \times 100 \%$ was used, where the $C_{0}$ is the initial MB concentration $(\mathrm{t}=0 \mathrm{~h})$, and $C$ is the remaining concentration of MB dye at any time, $t$. Results indicate that after $6 \mathrm{~h}$ of UV-C exposure, all ZnO NPs show a photodegradation activity higher than $40 \%$, as shown in Fig. $7(\mathrm{~d})$, being the highest activity near to 100 \% for the $\mathrm{ZnO}$ NPs annealed at $700{ }^{\circ} \mathrm{C}$. It is good mentioning that the MB dye solution without $\mathrm{ZnO}$ NPs shows 
photodegradation of less than $1 \%$ after $6 \mathrm{~h}$ of UV-C exposure, which confirms the PCA of the $\mathrm{ZnO}$ nanopowders. To compare the performance of the $\mathrm{ZnO}$ NPs annealed at different temperatures, we estimated the remaining $\mathrm{MB}$ concentration after $6 \mathrm{~h}$ of light exposure for all the studied samples and the results are shown in Fig. 7 (e). As observed, photodegradation activity is $\sim 45 \%$ for the sample annealed at $500{ }^{\circ} \mathrm{C}$. Increasing the annealing temperature leads to the increase of the photodegradation activity, reaching the highest performance $(\sim 100 \%)$ for the sample annealed at $700{ }^{\circ} \mathrm{C}$. Above the annealing temperature of $700{ }^{\circ} \mathrm{C}$, the photodegradation activity shows a decreasing tendency until it reaches the value of $\sim 70 \%$, obtained for the sample annealed at 900 ${ }^{\circ} \mathrm{C}$. In order to corroborate our findings, a second set of samples was produced using the same annealing temperatures. Results of the PCA for the new set of samples provide a similar trend as the first set of samples. Our findings regarding the photodegradation activity strongly suggest that the PCA does not show a direct dependence on the surface area, once the surface area shows a decreasing trend with the annealing temperature, as evidenced above by the increase of the crystallite mean size with the annealing temperature. Similar findings were reported by Mekasuwandumrong et al. [23], which reported a maximum degradation value for intermediate particles sizes of ZnO NPs synthesized by spray flame reactor (authors worked with temperatures below $900{ }^{\circ} \mathrm{C}$ ). Verma et al. [24] also showed a photodegradation of direct red 31 (DR-31) dye using ZnO NPs annealed with temperatures in the range between 500 and $800^{\circ} \mathrm{C}$, obtaining the better PCA efficiency with the sample annealed at intermediate temperatures $\left(600-700^{\circ} \mathrm{C}\right)$ [25]. Also, Montero-Muñoz [26] determined that the PCA efficiency of $\mathrm{ZnO}$ on $\mathrm{MB}$ molecules is related to the morphology of the $\mathrm{ZnO}$ nanostructures more than the total surface area of the $\mathrm{ZnO}$ NPs. Therefore, it is clear that there is no direct relationship between the MB dye degradation and the particle size. Moreover, it is known that intrinsic defects, such as $\mathrm{Zn}$ vacancies $\left(\mathrm{V}_{\mathrm{Zn}}\right), \mathrm{Zn}$ interstitial $\left(\mathrm{Zn}_{\mathrm{i}}\right)$, oxygen interstitial $\left(\mathrm{O}_{\mathrm{i}}\right)$, and oxygen vacancies $\left(\mathrm{V}_{\mathrm{O}}\right)$ can exist in a $\mathrm{ZnO}$ structure. Depending on their density, the charge carrier concentration can change and, consequently, it can tune the PCA performance. In this regard, Janotti et al. [13] reported that depending on the annealing temperature, the intrinsic defects can be wiped out. At low temperatures (below $200{ }^{\circ} \mathrm{C}$ ) defects such as Frenkel pairs $\left(\mathrm{Zn}_{\mathrm{i}}+\mathrm{V}_{\mathrm{Zn}}\right), \mathrm{Zn}_{\mathrm{i}}{ }^{2+}, \mathrm{O}_{\mathrm{i}}{ }^{0}$, and $\mathrm{O}_{\mathrm{i}}{ }^{2-}$ are expected to be removed. Meanwhile, for intermediate temperatures, in the range between $200{ }^{\circ} \mathrm{C}$ and 300 
${ }^{\circ} \mathrm{C}$, and between $300{ }^{\circ} \mathrm{C}$ and $400{ }^{\circ} \mathrm{C}, \mathrm{V}_{\mathrm{Zn}}{ }^{2-}$ and the $\mathrm{V}_{\mathrm{O}}{ }^{2+}$ defects can be respectively annihilated. At high temperatures $\left(\sim 636{ }^{\circ} \mathrm{C}\right)$ the $\mathrm{V}_{0}$ are expected to be wiped out. In this scenery, a plausible explanation for the PCA changes as the annealing temperature is increased seems to be the remotion of crystal defects which can be wiped out from the crystal in a specific range of temperature. The obtained results suggest that when the annealing temperature is increased up to $700{ }^{\circ} \mathrm{C}$, the PCA is improved. Above that annealing temperature, the PCA tends to decrease, that seems to be related to the annihilation of oxygen vacancies, which is expected to occurs at $\sim 636{ }^{\circ} \mathrm{C}$, according to the literature [11].

To study the kinetics of the PCA, the pseudo-first-order reaction described in the LangmuirHinshelwood mechanism was used [8]:

$$
\ln \left(C_{0} / C\right)=K \cdot t
$$

here $K$ is the photodegradation rate constant and $t$ is the time. A linear trend of $\ln \left(C_{0} / C\right)$ with $t$ is determined, as exemplified for the sample annealed at $900{ }^{\circ} \mathrm{C}$ in the inset of Fig. 6 (f). In the main panel of Fig. 6 (f) one shows the dependence of $K$ with the annealing temperature. It is observed that $K$ increases with the increase of the annealing temperature reaching a maximum value for the sample annealed at $700{ }^{\circ} \mathrm{C}$, decreasing afterwards for higher annealing temperatures. 

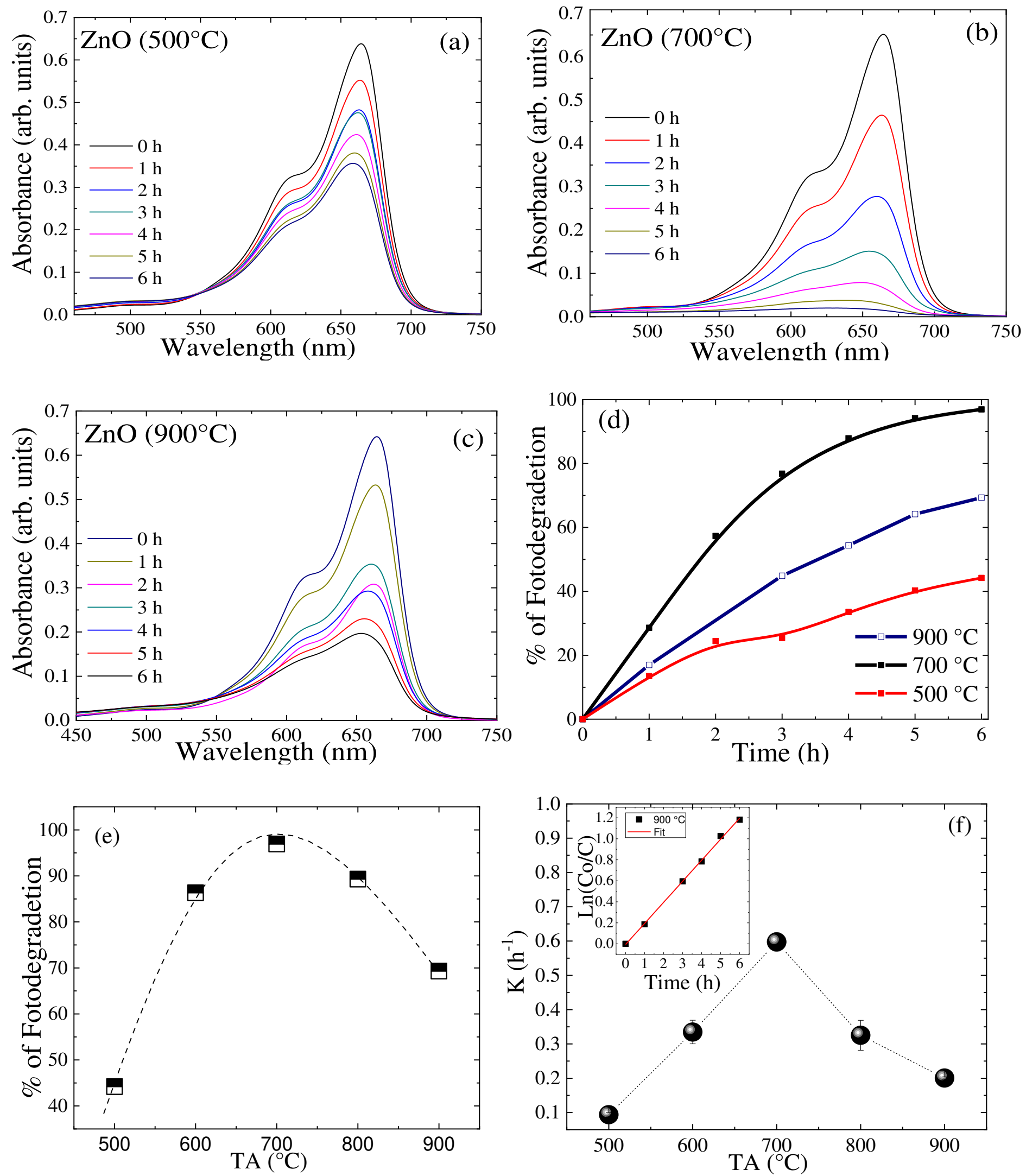

Figure 7 UV-vis spectra of MB dye solution containing ZnO NPs annealed at (a) $500{ }^{\circ} \mathrm{C}$, (b) $700{ }^{\circ} \mathrm{C}$, and (c) $900{ }^{\circ} \mathrm{C}$ as a function of the UV-C exposure time. (d) MB photodegradation percentage as a function of time for 
three annealed samples. (e) MB photodegradation percentage as a function of annealing temperature. (f) Dependence of the photodegradation rate constant on the annealing temperature. The inset shows the linear trend of $K$ determined from the pseudo-first-order reaction.

\subsection{Thermoluminescence study}

Once $\mathrm{ZnO}$ is known as a thermoluminescent material [14], the thermoluminescence (TL) properties can be used to determine the activation energy of traps inside the band gap. It is known that during the exposure to ionization irradiation (such as gamma or UV-C irradiation) the material absorbs energy proportional to the energy of the trap, which is released as visible light during the material heating. TL glow-curves were collected for all samples. Figure 8 shows the TL curves of the $\mathrm{ZnO}$ nanopowders annealed at different temperatures and exposed to $1 \mathrm{~Gy}$ of gamma radiation from a ${ }^{60} \mathrm{Co}$ source. A strong browned TL glow peak at $622 \mathrm{~K}$, as well as a tiny peak at $380 \mathrm{~K}$, are observed for the as-prepared sample (see Fig. 8 (a)). Actually, the prominent glow peak observed in the as-prepared sample is observed in all samples; however, in sample annealed at $900{ }^{\circ} \mathrm{C}$ other two high intensity overlapping peaks are found around 450 and $525 \mathrm{~K}$, which are associated with the increase of the sample's crystallinity [27]. Generally, the glow peak is composed of several individual TL peaks [14]. However, to obtain an average behavior, the browned peak at $622 \mathrm{~K}$ is fitted assuming only one peak using the least-squares Levenberg-Marquardt method implemented in the GlowFit program. In this case, the thermoluminescence intensity $I(T)$ of the glow peak is given by the Randall-Wilkins second-order expression of a single glow peak [27] [28]. This is due to the almost symmetrical shape of the considered TL peak. The second-order glow-curves appear due to the higher probability of retrapping as compared to the first-order case [29]:

$$
I(T)=I_{m} \exp \left(\frac{E}{K T_{m}}-\frac{E}{K T}\right) \exp \left(-\frac{E}{K T_{m}^{2}} \int_{T_{m}}^{T} \exp \left(\frac{E}{K T_{m}}-\frac{E}{K T^{\prime}} d T^{\prime}\right)\right)
$$

where $T_{m}$ and $I_{m}$ are the temperature and the intensity of the maximum, respectively. $E$ in Eq. (5) is the activation energy in $\mathrm{eV}$, and $K$ is the Boltzmann constant. Using Eq. (5) to fit the TL curves in Figs. 8 (a)-(c), 
the temperature of the peak at its maximum and the corresponding intensity were determined. For the asprepared $\mathrm{ZnO} \mathrm{NPs}$, the $T_{m}$ is found at $622 \mathrm{~K}$. Moreover, the values found for $T_{m}$ show a shift to higher temperatures as the annealing temperature increases (see left-hand side axis in Fig. 8 (d)). Additionaly, an interesting finding is that the integrated intensity of the high-temperature glow peak displays a decreasing tendency as the annealing temperature increases, showing a minimum value at $700{ }^{\circ} \mathrm{C}$ (see right-hand side axis in Fig. 8 (d)). The latter result suggests that the content of TL traps becomes smaller when the ZnO samples are subjected to higher annealing temperatures, reaching the lowest content for the sample annealing at $700{ }^{\circ} \mathrm{C}$. Above that annealing temperature, a slight increase of the integrated intensity is observed. This result could be directly related to the number of electron-holes produced during the UV-C radiation exposure, which can be trapped in the TL traps. It means that in samples presenting a large number of traps, part of the UV-C radiation is absorbed and stored in TL traps, decreasing the PCA; meanwhile, in the nanopowders with a lower content of TL traps, more UV-C radiation can participate effectively in the photocatalytic degradation. Furthermore, as the annealing temperature is increased, larger activation energy is determined, which could be associated with the remaining defects, mostly oxygen vacancies appearing as the material improves its crystallinity, which show higher annihilation energy. 

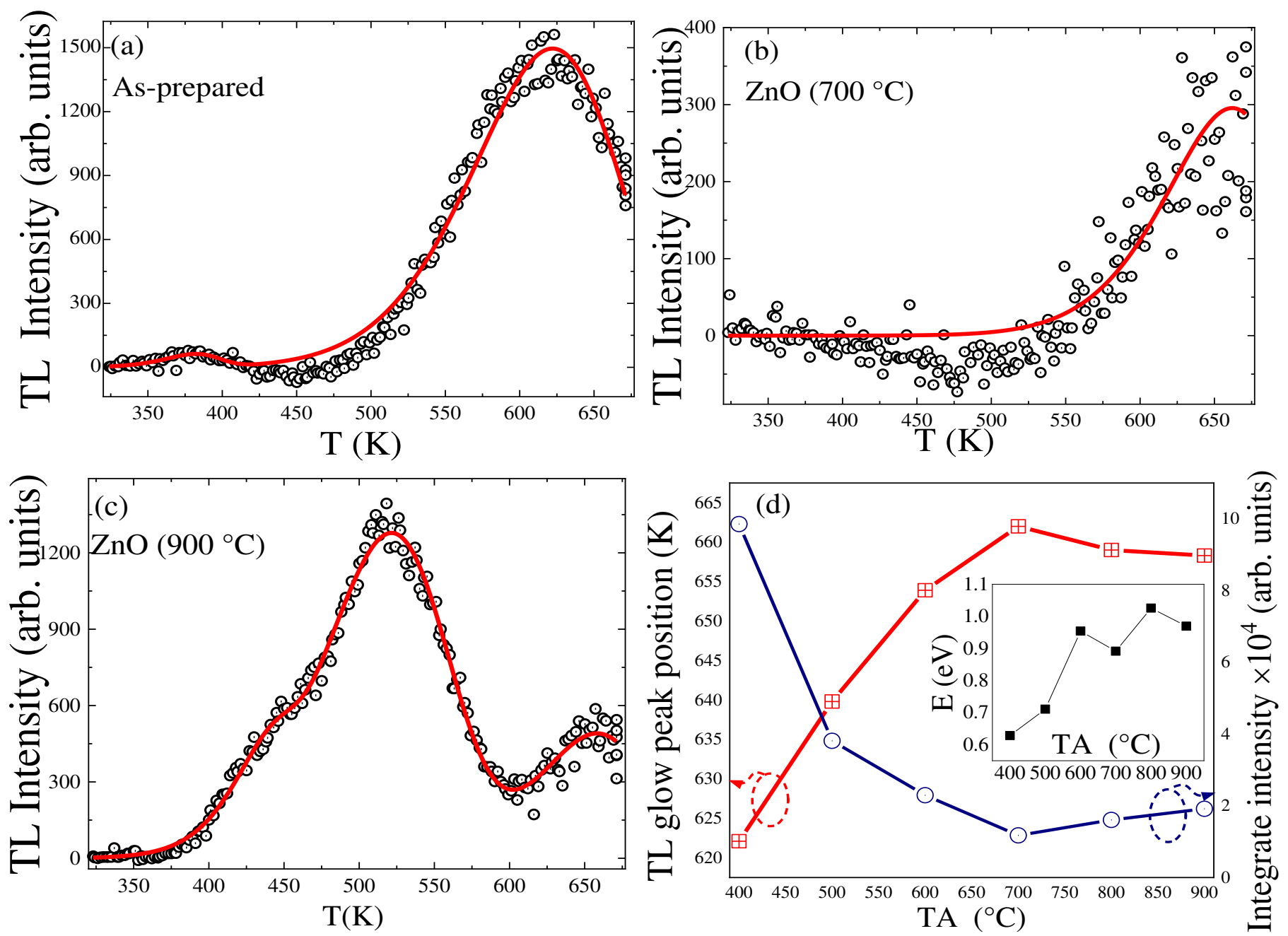

Figure 8 Glow curves of $\mathrm{ZnO}$ nanopowders sintered at different temperatures and after being exposed to $1 \mathrm{~Gy}$ of gamma irradiation.

\section{Conclusions}

Thermogravimetric analysis (TGA) measurement performed in the polymeric precursor of $\mathrm{ZnO}$ nanoparticles (NPs) shows three distinct weight-loss steps related to the elimination of adsorbed water and ethylene glycol; combustion of molecular species such as citric acid and nitrate; and formation of stable $\mathrm{ZnO}$ NPs above $\sim 400{ }^{\circ} \mathrm{C}$. Before and after MB dye degradation, x-ray diffraction patterns of the as-fabricated nanopowders exhibited no structural changes due to physical interaction between the NPs and the dye molecules, implying that the nanopowders can be reused. Furthermore, the effect of the thermal treatment is evidenced by a change in the $c / a$ lattice parameters ratio plus the increase of the mean crystallite size. The latter 
exhibits a noticeable monotonic lowering of the optical band gap, which is strongly correlated with the samples' photocatalic activity (PCA). FTIR measurements are in very good agreement with the XRD patterns, with no change of the wurtzite $\mathrm{ZnO}$ structure after the $\mathrm{MB}$ degradation. The data herein reported suggest that the dependence of particle size with PCA is not straightforward. Although reduction of the particle size implies the increase of the total surface area, it seems that this is not the main reason for enhancement of the PCA. Likewise, the type and the content of intrinsic defects in the nanopowder samples are not directly correlated with the mean crystallite size. Moreover, the data herein presented show that the type and the amount of defects can be modulated by thermal annealing. In this regard, our findings suggest that the PCA of the fabricated $\mathrm{ZnO}$ nanopowders, for degrading methylene blue dye, seems to be correlated to the density of defects, such as oxygen vacancies, whose content is modulated by the annealing temperature. Finally, the thermoluminescence glow curves proved to be a powerful tool for determining the PCA of nanomaterials, once they are directly related to the density of trapped charges (electrons/holes).

\section{Acknowledgments}

The authors (FFHA, JCRA, JAHC, JLS, and DGPS) thank the financially supported by CONCYTECFONDECYT within the framework of the call E038-01 [contract No 07-2019-FONDECYT-BM-INC. INV]. JAHC thanks the financial support from the Brazilian agencies CNPq (303182/2020-2) and FAPDF (0019300000151/2019-20). 


\section{References}

[1] D. A. Yaseen and M. Scholz, "Textile dye wastewater characteristics and constituents of synthetic effluents: a critical review," International Journal of Environmental Science and Technology, vol. 16, pp. 1193-1226, 2019/02/01 2019.

[2] K. Paździor, L. Bilińska, and S. Ledakowicz, "A review of the existing and emerging technologies in the combination of AOPs and biological processes in industrial textile wastewater treatment," Chemical Engineering Journal, vol. 376, p. 120597, 2019/11/15/ 2019.

[3] N. R. J. Hynes, J. S. Kumar, H. Kamyab, J. A. J. Sujana, O. A. Al-Khashman, Y. Kuslu, A. Ene, and B. Suresh Kumar, "Modern enabling techniques and adsorbents based dye removal with sustainability concerns in textile industrial sector -A comprehensive review," Journal of Cleaner Production, vol. 272, p. $122636,2020 / 11 / 01 / 2020$.

[4] V. Srikant and D. R. Clarke, "On the optical band gap of zinc oxide," Journal of Applied Physics, vol. 83, pp. 5447-5451, 1998/05/15 1998.

[5] J. Huang, Z. Yin, and Q. Zheng, "Applications of ZnO in organic and hybrid solar cells," Energy \& Environmental Science, vol. 4, pp. 3861-3877, 2011.

[6] Y. Kang, F. Yu, L. Zhang, W. Wang, L. Chen, and Y. Li, "Review of ZnO-based nanomaterials in gas sensors," Solid State Ionics, vol. 360, p. 115544, 2021/02/01/ 2021.

[7] A. P. Piedade, A. C. Pinho, R. Branco, and P. V. Morais, "Evaluation of antimicrobial activity of ZnO based nanocomposites for the coating of non-critical equipment in medical-care facilities," Applied Surface Science, vol. 513, p. 145818, 2020/05/30/2020.

[8] A. Di Mauro, M. E. Fragalà, V. Privitera, and G. Impellizzeri, "ZnO for application in photocatalysis: From thin films to nanostructures," Materials Science in Semiconductor Processing, vol. 69, pp. 44-51, 2017/10/01/ 2017. 
[9] S. Kuriakose, B. Satpati, and S. Mohapatra, "Enhanced photocatalytic activity of Co doped ZnO nanodisks and nanorods prepared by a facile wet chemical method," Physical Chemistry Chemical Physics, vol. 16, pp. 12741-12749, 2014.

[10] M. Montero-Muñoz, J. E. Ramos-Ibarra, J. E. Rodríguez-Páez, M. D. Teodoro, G. E. Marques, A. R. Sanabria, P. C. Cajas, C. A. Páez, B. Heinrichs, and J. A. H. Coaquira, "Role of defects on the enhancement of the photocatalytic response of $\mathrm{ZnO}$ nanostructures," Applied Surface Science, vol. 448, pp. 646-654, 2018/08/01/ 2018.

[11] M. Montero-Muñoz, J. E. Ramos-Ibarra, J. E. Rodríguez-Páez, G. E. Marques, M. D. Teodoro, and J. A. H. Coaquira, "Growth and formation mechanism of shape-selective preparation of $\mathrm{ZnO}$ structures: correlation of structural, vibrational and optical properties," Physical Chemistry Chemical Physics, vol. 22, pp. 7329-7339, 2020.

[12] F. Kayaci, S. Vempati, I. Donmez, N. Biyikli, and T. Uyar, "Role of zinc interstitials and oxygen vacancies of $\mathrm{ZnO}$ in photocatalysis: a bottom-up approach to control defect density," Nanoscale, vol. 6, pp. 10224-10234, 2014.

[13] A. Janotti and C. G. Van de Walle, "Native point defects in ZnO," Physical Review B, vol. 76, p. $165202,2007$.

[14] H. A. Borbón-Nuñez, C. Cruz-Vázquez, R. Bernal, G. Kitis, C. Furetta, and V. M. Castaño, "Thermoluminescence properties of sintered ZnO," Optical Materials, vol. 37, pp. 398-403, 2014/11/01/ 2014.

[15] B. Toby, "EXPGUI, a graphical user interface for GSAS," Journal of Applied Crystallography, vol. 34, pp. 210-213, 2001.

[16] A. Galdámez-Martinez, G. Santana, F. Güell, P. R. Martínez-Alanis, and A. Dutt, "Photoluminescence of ZnO Nanowires: A Review," Nanomaterials, vol. 10, p. 857, 2020.

[17] M. Pudukudy and Z. Yaakob, "Facile Synthesis of Quasi Spherical ZnO Nanoparticles with Excellent Photocatalytic Activity," Journal of Cluster Science, vol. 26, pp. 1187-1201, 2015/07/01 2015. 
[18] J. Wang, Z. Wang, B. Huang, Y. Ma, Y. Liu, X. Qin, X. Zhang, and Y. Dai, "Oxygen Vacancy Induced Band-Gap Narrowing and Enhanced Visible Light Photocatalytic Activity of ZnO," ACS Applied Materials \& Interfaces, vol. 4, pp. 4024-4030, 2012/08/22 2012.

[19] Z.-Y. Zhang, "Strain engineering for ZnO nanowires: First-principle calculations," Physics Letters A, vol. 378, pp. 1174-1179, 2014/03/14/ 2014.

[20] R. Raji and K. G. Gopchandran, "ZnO nanostructures with tunable visible luminescence: Effects of kinetics of chemical reduction and annealing," Journal of Science: Advanced Materials and Devices, vol. 2, pp. 51-58, 2017/03/01/ 2017.

[21] N. Jayarambabu, Kumari, K. Rao, and Y. Prabhu, "BENEFICIAL ROLE OF ZINC OXIDE NANOPARTICLES ON GREEN CROP PRODUCTION," INTERNATIONAL JOURNAL OF MULTIDISCIPLINARY ADVANCED RESEARCH TRENDS, vol. 2, pp. 2349-7408, 2015.

[22] D. Heger, J. Jirkovský, and P. Klán, "Aggregation of Methylene Blue in Frozen Aqueous Solutions Studied by Absorption Spectroscopy," The Journal of Physical Chemistry A, vol. 109, pp. 6702-6709, 2005/08/04 2005 .

[23] O. Mekasuwandumrong, P. Pawinrat, P. Praserthdam, and J. Panpranot, "Effects of synthesis conditions and annealing post-treatment on the photocatalytic activities of $\mathrm{ZnO}$ nanoparticles in the degradation of methylene blue dye," Chemical Engineering Journal, vol. 164, pp. 77-84, 2010/10/15/ 2010.

[24] N. Verma, S. Bhatia, and R. K. Bedi, "Effect of Annealing Temperature on ZnO Nanoparticles and its Applications for Photocatalytic Degradation of DR- 31 dye," International Journal of Pure and Applied Physics. ISSN 0973-1776, vol. 13, pp. 118-122, 2017.

[25] T. Ivetic, M. Dimitrievska, N. Finčur, L. Đačanin, I. O. Gúth, B. Abramović, and S. Lukic-Petrovic, "Effect of Annealing Temperature on Structural and Optical Properties of Mg-Doped ZnO Nanoparticles and Their Photocatalytic Efficiency in Alprazolam Degradation," Ceramics International, vol. 40, pp. $1545-1552,2014$. 
[26] M. Montero-Muñoz, "Estudo dos mecanismos de formação de estruturas de ZnO com diferente morfologia e a sua influência na eficiência da atividade fotocatalítica sobre moléculas orgânicas," PhDThesis, University of Brasilia, 2018.

[27] M. Puchalska and P. Bilski, "GlowFit - a new tool for thermoluminescence glow-curve deconvolution," Radiation Measurements, vol. 41, pp. 659-664, 2006/07/01/ 2006.

[28] A. J. J. Bos, "Theory of thermoluminescence," Radiation Measurements, vol. 41, pp. S45-S56, 2006/12/01/ 2006.

[29] S. W. S. McKeever, "Thermoluminescence of Solids," Cambridge Uni. Press., Cambridge, 1985. 